\title{
Perioperative Microcirculatory Changes Detected with Gastroscopy Assisted Laser Doppler Flowmetry and Visible Light Spectroscopy in Patients with Median Arcuate Ligament Syndrome
}

This article was published in the following Dove Press journal:

Vascular Health and Risk Management

\author{
Simen Tveten Berge' \\ Nathkai Safi ${ }^{2}$ \\ Asle W Medhus $\mathbb{D}^{3}$ \\ Jon $O$ Sundhagen ${ }^{1}$ \\ Jonny Hisdal ${ }^{1,2}$ \\ Syed SH Kazmi ${ }^{1,2}$ \\ 'Department of Vascular Surgery, Oslo \\ University Hospital HF, Oslo, Norway; \\ ${ }^{2}$ Faculty of Medicine, University in Oslo, \\ Oslo, Norway; ${ }^{3}$ Department of \\ Gastroenterology, Oslo University \\ Hospital HF, Oslo, Norway
}

Purpose: Physiological tests may aid in diagnosing median arcuate ligament syndrome (MALS). MALS is a symptomatic compression of the celiac artery causing symptoms similar to chronic mesenteric ischemia (CMI) of atherosclerotic etiology. Simultaneous use of visible light spectroscopy (VLS) and laser doppler flowmetry (LDF) during upper endoscopy may detect microcirculatory changes in these patients.

Patients and Methods: In a single-center, prospective comparative cohort, 25 patients were evaluated for MALS. Patients with a consensus diagnosis of MALS $(n=15)$ underwent a gastroscopy assisted, transmucosal microcirculatory assessment with LDF and VLS. Results were compared to individuals with normal intestinal circulation $(n=38)$ evaluated with duplex ultrasonography, and to patients with chronic mesenteric ischemia $(n=32)$. Treatment response was evaluated clinically at 1, 3, 6, and 12 months, and with ultrasound, VLS and LDF at three months. Health-related quality of life (QoL) was assessed with Euroqol (EQ-5D-5L), preoperatively, and 12 months postoperatively.

Results: Preoperative mean transmucosal oxygen saturation was significantly lower in patients with MALS $\left(\mathrm{SO}_{2} 76 \pm 6\right)$, as compared to healthy individuals $\left(\mathrm{SO}_{2} 81 \pm 4\right), \mathrm{p}=0.02$. An overall significant improvement in $\mathrm{SO}_{2}$ after surgical decompression of the celiac artery was found $\left(\mathrm{SO}_{2} 81 \pm 3.7, \mathrm{p}=0.05\right)$. Eleven $(92 \%)$ patients with clinical improvement after laparoscopic decompression had a definitive diagnosis of MALS. Median follow-up was 18 months (4-24 months). Four of the five dimensions investigated with EQ-5D-5L improved. Conclusion: VLS detected a significantly lower baseline transmucosal $\mathrm{SO}_{2}$ in patients with MALS as compared to control subjects with normal intestinal circulation. An improvement in $\mathrm{SO}_{2}$ after laparoscopic decompression was found, supporting a possible ischemic etiology in our patient population.

Keywords: mesenteric ischemia, functional test, endoscopy, vascular surgery, abdominal pain

\section{Introduction}

Median arcuate ligament syndrome (MALS) is caused by compression of the celiac artery (CA) by the median arcuate ligament and was first described anatomically by Lipschutz in 1917. ${ }^{1}$ The median arcuate ligament is a fibrous band that crosses over the aorta and connects the right and left crura of the diaphragm. The anatomical variant with compression of the CA due to either a high origin of the artery or a low insertion of the crura is present in $10-24 \%$ of the population. ${ }^{2,3}$ This is the most

Syed SH Kazmi

Department of Vascular Surgery, Oslo

University Hospital HF, Oslo 0586,

Norway

Tel +4792468309

Email sshkazmi@gmail.com 
common cause of single-vessel stenosis in the mesenteric arteries. Although this external compression is usually asymptomatic, it can, in some patients, cause postprandial epigastric pain or nausea, weight loss, and an epigastric bruit. $^{2,4}$

The diagnosis is controversial, mainly because the pathophysiology of MALS remains unclear. The prevailing theory is that insufficient amounts of blood pass through the compressed CA in times of increased metabolic demand, leading to ischemia., ${ }^{4,5}$ Another theory is that a neuropathic compression of the vessel may lead to irritation of sympathetic pain fibers causing pain, much like in the case of other nerve compression diseases, such as a carpal tunnel. ${ }^{6}$

The clinical presentation of MALS is variable. ${ }^{4}$ The diagnosis depends on exclusion of other possible causes of abdominal pain, and patients often undergo extensive examinations. Upper endoscopy is a standard initial investigation in patients with postprandial upper abdominal complaints. ${ }^{4,7}$ Using gastric exercise tonometry (GET) as a functional test, one study has demonstrated ischemia in patients with MALS. ${ }^{8}$ The test is, however, somewhat cumbersome, the required equipment is no longer available, and alternative functional tests hence need to be explored.

Gastroscopy assisted laser Doppler flowmetry (LDF) and visible light spectroscopy (VLS) (GALS) have successfully been utilized to examine transmucosal microcirculatory changes in the gastrointestinal tract both in healthy individuals and patients with chronic mesenteric ischemia (CMI) due to atherosclerosis of the mesenteric arteries. ${ }^{9,10}$ Even in the case of a single vessel pathology, GALS has been able to detect ischemic changes in the stomach and duodenum. ${ }^{10,11}$

Traditionally, the treatment of MALS has been an open surgical release of the compression on the celiac artery, but for the last decade, a laparoscopic approach has been utilized. $^{12,13}$

This study aimed to examine the transmucosal microcirculation of the stomach and duodenum in the patients with MALS utilizing GALS before and after the laparoscopic decompression of the CA.

We hypothesized that patients with MALS have reduced microcirculation in the stomach as compared to subjects with healthy intestinal circulation and that the microcirculation and symptoms improve in patients after the surgical procedure. Furthermore, we hypothesized that the patient's health-related quality of life (QoL) improved after surgery.

\section{Patients and Methods}

The study was designed as a single-center prospective comparative cohort. It was conducted at Oslo University Hospital during a 36 months period from September 2016 to September 2019. Patients included were referred from either primary health care or other hospitals. The suspicion of MALS was based on symptomatology, CT angiography findings ( $\geq 50 \%$ stenosis in deep expiration phase (Figure 1)), and exclusion of common differential diagnoses such as ulcer disease, cholelithiasis, pancreatitis, inflammatory bowel disease, and malignancy. All patients were subjected to a detailed clinical examination and CT angiography before discussion in a multidisciplinary panel comprising of vascular surgeons and interventional radiologists. Patients were required to have at least two of the three following criteria; postprandial pain, unintentional weight loss, and changes in habits of food intake (reduced amount and/or increased frequency of meals, the exact frequency and composition of each meal was not recorded). CT angiography in deep inspiration and deep expiration phase was undertaken. A reconstruction in axial, sagittal, and coronal planes was examined to confirm

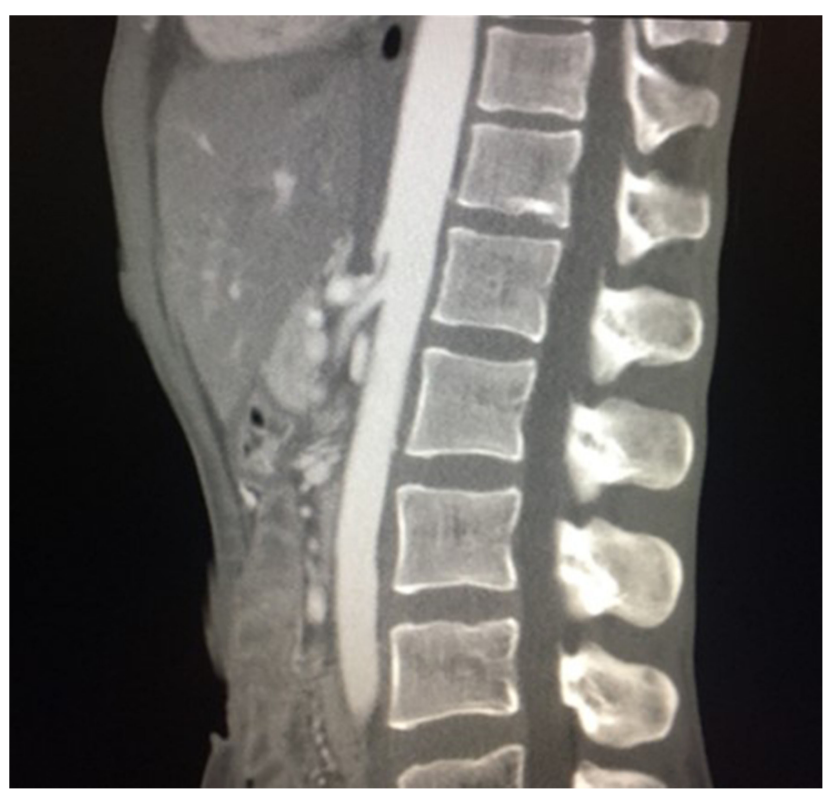

Figure I CTA sagittal plane of a patient with MALS taken in deep expiration. An external compression of the celiac artery and a normal anatomy of the superior mesenteric artery is demonstrated.

Abbreviations: MALS, median arcuate ligament syndrome; CTA, computed tomography angiography. 
MALS. A consensus diagnosis of MALS was made based on the above.

In patients with a consensus diagnosis of MALS, a transmucosal microcirculatory investigation with GALS was performed. The investigation was repeated after surgical treatment, and results were compared to data from individuals with healthy intestinal circulation (Control Group 1 (CG1) $\mathrm{n}=38$ ). The individuals in CG1 were recruited from a list of patients awaiting upper endoscopy due to dyspepsia, control of Barrett's esophagus, or controls after ulcer disease. None of these had postprandial abdominal pain or weight loss. A transabdominal duplex ultrasound (Vivid E95, General Electric Healthcare, Chicago, IL) with a curvilinear probe $\mathrm{C} 1-6$ was performed after a minimum of six hours fasting to evaluate the patency of the intestinal arteries in these control individuals. An experienced specialist in ultrasonography performed all examinations. Significant stenosis $(>70 \%)$ was defined as a peak systolic velocity of the superior mesenteric artery (SMA) $\geq 275 \mathrm{~cm} / \mathrm{s}$ and CA $\geq 200 \mathrm{~cm} / \mathrm{s}^{14}$

Additionally, data from MALS patients were compared to previously published data on patients with chronic mesenteric ischemia (CMI), (Control Group 2 (CG2), $\mathrm{n}=32$ ) due to atherosclerosis. ${ }^{10}$ In this previous study, the inclusion criterion was stenosis $\geq 70 \%$ or occlusion of $\geq 1$ mesenteric artery on CT angiography in addition to symptom relief after intervention with percutaneous transluminal angioplasty with or without stenting or aortomesenteric bypass.

Symptom relief was defined as either a complete or partial disappearance of symptoms. A definitive diagnosis of MALS was thereby based on a complete or partial relief of symptoms after an intervention.

\section{Measurements}

All patients were investigated with upper endoscopy at the gastric laboratory before and three months after the laparoscopic surgical treatment. All endoscopies were performed by the same team of examiners, comprising an experienced gastroenterologist and two vascular surgeons. The patients were examined in a fasting state and in a left, lateral decubitus position. Peripheral oxygen saturation was monitored in all patients receiving benzodiazepines or analgesics and kept $>95 \%$. Air insufflation was kept at a minimum, and no spasmolytic agent was administered.

The measurement points were chosen based on the arterial supply of the stomach and duodenum by the branches of the CA and superior mesenteric artery (SMA)

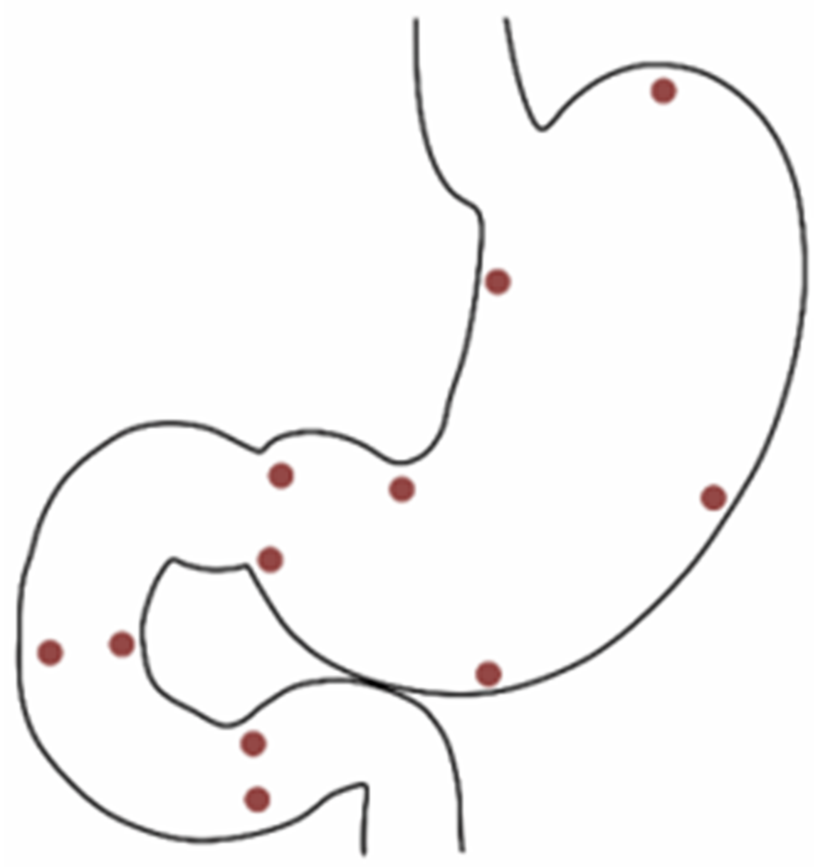

Figure 2 Measurement points in the stomach and duodenum in the study of perioperative microcirculatory changes in patients with MALS.

Abbreviation: MALS, median arcuate ligament syndrome.

(Figure 2). A mean of the four measurements in the duodenum (descending and horizontal part), the four measurements at the lesser curvature and pylorus, and three measurements at the greater curvature were calculated.

A $2.6 \mathrm{~mm}$ "Oxygen 2 See" (O2C) microprobe, LM-10 (O2C, LEA Medizintechnik, Giessen, Germany), was passed through the working channel of an Olympus flexible gastroscope. The O2C utilizes both LDF and VLS to make simultaneous measurements of flow (i.e., red blood cell flux), velocity, combined venous and arterial saturation of capillary hemoglobin $\left(\mu \mathrm{Hb}_{\mathrm{SO} 2}\right)$ and a relative hemoglobin-amount $\left(\mu \mathrm{Hb}_{\text {con }}\right){ }^{15}$

The machine detected movement and pressure artifacts, and visually assessed unstable, or fluctuating recordings were discarded, and the measurements were automatically or manually repeated.

The study patients were followed up at 1 (clinical evaluation), 3 (upper endoscopy with microcirculatory measurements, clinical evaluation, duplex ultrasound), 6 (clinical evaluation), 12 (clinical evaluation, QoL assessment) months, and yearly (clinical evaluation) thereafter.

\section{Health-Related Quality of Life}

At inclusion and 12 months postoperatively, patients completed a validated and Norwegian translated questionnaire 
EuroQol (EQ-5D-5L). ${ }^{16}$ EQ-5D provides a descriptive profile and a single index value for health status based on five dimensions; mobility, self-care, usual activities, pain/discomfort, and anxiety/depression. Each of the five dimensions is divided into five levels of perceived problems from 1 (indicating no problems) to 5 (indicating extreme problems). The second part of the EQ-5D-5L is a visual analog scale from 0 (worst imaginable health state) to 100 (best imaginable health state).

\section{Operative Technique}

All patients were treated laparoscopically, and the same vascular surgeon performed all procedures. The patients were under general anesthesia and in a supine position with the surgeon placed between the legs of the patient. A $12 \mathrm{~mm}$ trocar was placed under visual guidance between the xiphoid process and umbilicus. The abdominal cavity was insufflated with $\mathrm{CO}_{2}$, three additional trocars $(5 \mathrm{~mm})$ were placed, one on the right side, and two on the left side of the patient's abdomen. The left liver lobe was elevated using Nathanson's liver retractor (Cook Medical, Bloomington, Indiana, United States). The omental bursa was opened through the division of the hepatogastric ligament. The common hepatic artery or splenic artery was identified and followed proximally to the celiac trunk bifurcation. The CA was dissected free using a monopolar hook until the median arcuate ligament was identified. The left gastric artery and the small diaphragmatic branches from the CA were preserved. The median arcuate ligament was divided with either the monopolar hook or Ultracision Harmonic (Ethicon Inc., Somerville, New Jersey, United States). Care was taken to clear the cranial surface of the CA and its origin from the aorta, from any fibrous, muscular or nervous tissue. The aorta was free dissected 2-3 cm cranially and on both sides of the origin of the celiac artery. After removing the Nathanson's liver retractor, exsufflation was done, and trocars were removed. Patients were discharged after a median hospital stay of 2 days (range 2-3 days).

\section{Ethics and Trial Registration}

Informed written consent was obtained from all patients and control subjects. The study protocol was approved by the Regional Committees for Medical and Health Research Ethics in the South-Eastern region of Norway (REK sør-øst B 2016/682) and registered in the ClinicalTrials.gov Protocol Registration and Results System (NCT02914912). The study was conducted in accordance with the Declaration of Helsinki.

\section{Statistics}

Normally distributed data are presented as mean values with standard deviations or median values with range unless otherwise stated. For continuous outcome variables, the independent Student's $t$-test was applied, and Fisher's exact test was applied to categorical data. Wilcoxon signed ranks test was used to investigate the change after intervention. The statistical significance was set at $5 \%$ $(\mathrm{p}<0.05)$.

A power analysis was performed based on the results from our previous study on CMI with a mean oxygen saturation of $81 \pm 4 \%$ in the individuals with healthy intestinal circulation and an anticipated saturation of $76 \%$ in patients with MALS (CMI 67 $\pm 9 \%$ ). With the study power set at $80 \%$, a sample size of 10 patients was calculated.

Test performance at different cut-off levels was explored using receiver operating characteristics (ROC) curve, and sensitivity and specificity for diagnosing MALS were calculated.

Statistical analysis was performed using IBM SPSS Statistics version 25 (IBM Corp. Armonk, NY).

\section{Results}

From September 2016 to September 2019, a total of 25 patients were referred from primary health care and from other hospitals to the Department of Vascular Surgery, Oslo University Hospital, for the evaluation of MALS (Figure 3). Of these, eight were excluded based on $\leq 50 \%$ stenosis on CTA in the deep expiration phase. Two had $\geq 50 \%$ stenosis, but no symptoms and the findings were regarded as incidental. Fifteen patients were hence included in this study, and based on the standard diagnostic workup, they were diagnosed with MALS and offered surgical treatment. Laparoscopic decompression was the treatment of choice in all patients; however, three patients chose to decline surgery and are being followed-up in the out-patient clinic. Twelve patients underwent a successful laparoscopic decompression of the CA. Of these, 11 reported clinical improvement on follow-up. These 11 patients have been considered as having a confirmed diagnosis of MALS and are included in the statistical analysis. Patient characteristics are presented in Table 1.

All patients with a confirmed diagnosis of MALS described postprandial abdominal pain upon referral, nine $(82 \%)$ of them reported pain debut within 30 minutes of 


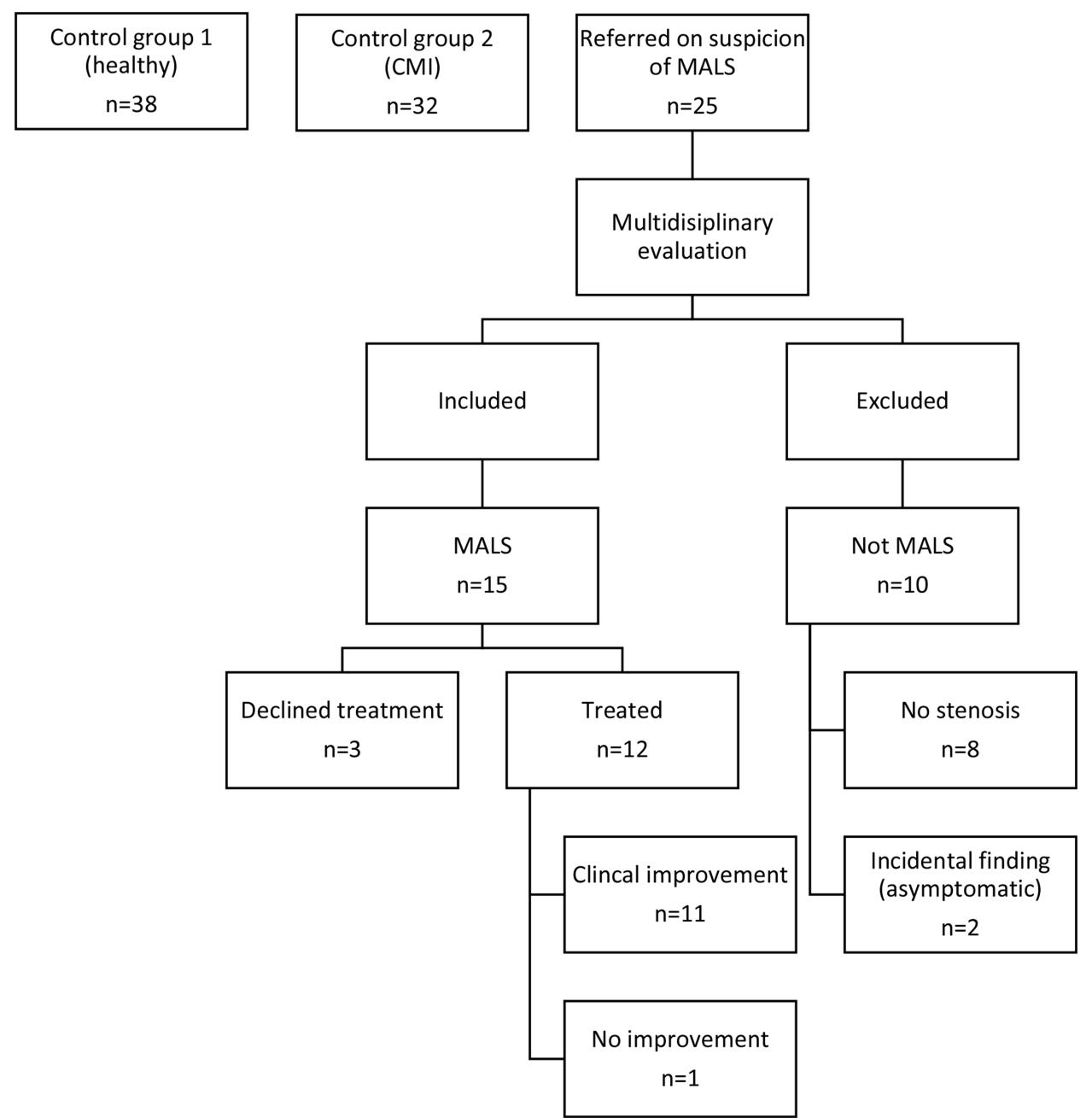

Figure 3 Flow chart of the inclusion process and outcomes in the study of perioperative microcirculatory changes in patients with MALS. Abbreviations: MALS, median arcuate ligament syndrome; CMI, chronic mesenteric ischemia.

a meal, two (18\%) experienced pain 30-60 minutes after a meal. The pain lasted for more than 60 minutes in six $(55 \%)$ of these patients. A mean weight loss of $5 \mathrm{~kg}( \pm 7 \mathrm{~kg})$ was reported in seven patients (64\%). The time from debut to diagnosis varied, with a median of 96 months (16-600). Baseline characteristics of CG1 and CG2 are presented in Table 2.

GALS could be performed in all patients with MALS. All endoscopies were negative with regard to the concomitant disease. The mean total examination time was 12 minutes ( \pm 6 minutes). Ten patients $(91 \%)$ agreed to go through a second upper endoscopy three months after surgical intervention. One successfully treated patient with symptom relief refused a second upper endoscopy due to discomfort during the endoscopic procedure.

The preoperative mean transmucosal oxygen saturation was significantly lower in patients with MALS $\left(\mathrm{SO}_{2} 76\right.$ $\pm 6 \%$ ), as compared to healthy individuals (CG1, $\mathrm{SO}_{2} 81$ $\pm 4 \%, p=0.02$, Table 3 , Figure 4 ). There was no statistically significant difference in relative $\mathrm{Hb}$ amount, flow, or velocity between the groups. The results of the preoperative measurements in CMI patients (CG2, $\mathrm{SO}_{2}$ 67士9\%) were, however, significantly lower than in both healthy individuals (CG1) and MALS patients $(\mathrm{p}<0.001, \mathrm{p}=0.004$ respectively).

Five patients with MALS (45\%) had oxygen saturation $\left(\mathrm{SO}_{2}\right)$ below the cut-off of $78 \%$ from our study on CMI. ${ }^{10}$ Using ROC analysis, we found the sensitivity for identifying an individual with MALS was 54\% and specificity $66 \%$ with $\mathrm{SO}_{2}$ cut-off of $78 \%$ (Figure 5). A cut-off of $80 \%$ increases the sensitivity to $82 \%$ with a specificity of $60 \%$.

After a median follow up of 18 months (4-24 months), eleven $(92 \%)$ of the 12 patients treated with laparoscopic 
Table I Patient Characteristics in the Study on Visible Light Spectroscopy and Laser Doppler Flowmetry During Upper Endoscopy in Patients with Median Arcuate Ligament Syndrome

\begin{tabular}{|l|l|}
\hline Patient Characteristics & $(\mathbf{n}=\mathrm{I} \mathrm{I})$ \\
\hline Reporting weight loss & $7(64)$ \\
Weight loss $(\mathrm{kg})^{\mathrm{a}}$ & $5 \pm 7$ \\
Abdominal pain & $\mathrm{II}(\mathrm{I00})$ \\
Debut $<30$ minutes after a meal & $9(82)$ \\
Debut $>30$ minutes after a meal & $2(18)$ \\
Duration of abdominal pain $<30$ minutes & $2(18)$ \\
Duration of abdominal pain $30-60$ minutes & $3(27)$ \\
Duration of abdominal pain $>60$ minutes & $6(55)$ \\
Diarrhea/obstipation/nausea & $9(8 \mathrm{I})$ \\
Changes in eating habits & $8(73)$ \\
Abdominal bruit & $8(73)$ \\
Time from debut until diagnosis (months) & $96(16-600)$ \\
\hline
\end{tabular}

Notes: Values are presented as ${ }^{\mathrm{a}}$ mean $\pm \mathrm{SD},{ }^{\mathrm{b}}$ median (range) or absolute numbers with percentages

decompression reported complete or partial improvement in their symptoms compared to pre-intervention status. A partial recurrence of preoperative complaints was reported in four patients $(36 \%)$. However, they still reported improvement in their symptoms compared with the preoperative status. All of these had an open CA on follow-up duplex ultrasound.
At three months, a second upper endoscopy was performed. An overall significant improvement in $\mathrm{SO}_{2}$ after surgical decompression of the $\mathrm{CA}$ was found (preoperative $\mathrm{SO}_{2} 76 \pm 6$, postoperative $\mathrm{SO}_{2} 81 \pm 3.7, \mathrm{p}=0.05$ ). On an individual basis, an improvement in transmucosal $\mathrm{SO}_{2}$ was found in eight (72\%) of the MALS patients postoperatively (Figure 6). Two patients had no change in saturation (including one patient without symptom relief). Both $\mathrm{rHb}$ and flow increased after intervention, whereas velocity declined (Table 4). With regards to velocity, there was an on average higher pre-interventional velocity in MALS patients compared to controls (CG1 and CG2) (Table 3). A reduction in velocity was observed postoperatively, however, not being statistically significant.

A normalization of peak systolic velocity on duplex ultrasound was found postoperatively in ten patients (Table 5). One had an open CA, but PSV $>2,0 \mathrm{~m} / \mathrm{s}$ postoperatively.

EQ5D-5L was completed pre- and postoperatively by 9 of the 11 successfully treated patients. The visual analog scale showed an overall improvement from a score of 44 preoperatively to 62 postoperatively. Improvement after surgery was recorded in all patients except one. Four of the five dimensions investigated with EQ-5D-5L

Table 2 Baseline Characteristics of Patients with MALS and Control Subjects with Normal Intestinal Circulation (CGI), and Control Subjects with Chronic Mesenteric Ischemia (CMI, CG2)

\begin{tabular}{|c|c|c|c|c|c|}
\hline \multirow[t]{2}{*}{ Variables } & MALS & CGI (Healthy) & p-value & CG2 (CMI) & \multirow[t]{2}{*}{ p-value } \\
\hline & $n=I I$ & $n=38$ & & $n=32$ & \\
\hline Age (years) & $45(24-72)$ & $60(20-82)$ & 0.01 & 73 (53-89) & $<0.001$ \\
\hline Height (cm) & $172 \pm 6$ & $169 \pm 9$ & 0.22 & $168 \pm 9$ & 0.20 \\
\hline Weight (kg) & $62 \pm 9$ & $74 \pm 16$ & 0.02 & $64 \pm 16$ & 0.89 \\
\hline BMI & $21 \pm 2$ & $26 \pm 5$ & 0.001 & $22 \pm 5$ & 0.43 \\
\hline Female & $7(63)$ & $2 I(55)$ & 0.73 & $20(63)$ & 0.52 \\
\hline Smoking history & $8(73)$ & $22(58)$ & 0.49 & $29(91)$ & 0.16 \\
\hline Ischemic heart disease & $0(0)$ & $7(18)$ & 0.33 & $\mid 3(4 \mid$ & 0.02 \\
\hline Atrial fibrillation & $0(0)$ & I (3) & I & $5(16)$ & 0.30 \\
\hline Stroke/TIA & $0(0)$ & $3(8)$ & I & $5(16)$ & 0.30 \\
\hline Diabetes mellitus & $0(0)$ & $5(13)$ & 0.57 & $12(38)$ & 0.02 \\
\hline Hypertension & $\mathrm{I}(9)$ & $10(26)$ & 0.41 & $22(69)$ & 0.001 \\
\hline Lung disease & $2(18)$ & $6(16)$ & I & $7(22)$ & I \\
\hline Intermittent claudication & $0(0)$ & $0(0)$ & - & $12(38)$ & 0.02 \\
\hline \multicolumn{6}{|l|}{ Medication } \\
\hline Single platelet & $0(0)$ & $8(2 I)$ & 0.41 & $23(72)$ & $<0.001$ \\
\hline Double platelet & $0(0)$ & I (3) & 0.49 & $7(22)$ & 0.40 \\
\hline Statin & $0(0)$ & $10(26)$ & 0.15 & $28(88)$ & $<0.001$ \\
\hline
\end{tabular}

Note: Values Presented as Mean (SD) or Median (Range) or Absolute Numbers with Percentages.

Abbreviations: MALS, median arcuate ligament syndrome; CGI, control group I; CG2, control group 2. 
Table 3 Mean Values of Combined Arterial and Venous Oxygen Saturation $\left(\mathrm{SO}_{2}\right)$, Relative Hemoglobin Amount ( $\mathrm{rHb}$ ), Flow and Velocity for the Three Examined Areas in the Stomach and Duodenum, and a Mean of All Areas in Patients with MALS, CGI and CG2. $\mathrm{SO}_{2}$ in Percent. Relative Hemoglobin Amount, Flow and Velocity in Arbitrary Units

\begin{tabular}{|c|c|c|c|c|c|c|c|c|c|}
\hline \multirow[t]{2}{*}{ Variables } & & Duodenum & \multirow[t]{2}{*}{ p-value } & $\begin{array}{l}\text { Pylorus and } \\
\text { Lesser } \\
\text { Curvature }\end{array}$ & \multirow[t]{2}{*}{ p-value } & $\begin{array}{l}\text { Greater } \\
\text { Curvature }\end{array}$ & \multirow[t]{2}{*}{ p-value } & $\begin{array}{l}\text { Total } \\
\text { All Areas }\end{array}$ & \multirow[t]{2}{*}{ p-value } \\
\hline & & Mean士SD & & Mean士SD & & Mean士SD & & $\begin{array}{l}\text { Mean } \\
\pm S D\end{array}$ & \\
\hline \multirow[t]{3}{*}{$\mathrm{SO}_{2}$} & MALS $(n=I I)$ & $67 \pm 8$ & & $83 \pm 8$ & & $78 \pm 11$ & & $76 \pm 6$ & \\
\hline & CGI $(n=38)$ & $75 \pm 8$ & 0.006 & $84 \pm 6$ & 0.76 & $82 \pm 6$ & 0.13 & $8 I \pm 4$ & 0.02 \\
\hline & CG2 (CMI) $(n=32)$ & $57 \pm 14$ & 0.02 & $74 \pm 10$ & 0.01 & $70 \pm 12$ & 0.07 & $67 \pm 9$ & 0.004 \\
\hline \multirow[t]{3}{*}{$\mathrm{rHb}$} & MALS & $82 \pm 9$ & & $82 \pm 6$ & & $87 \pm 5$ & & $84 \pm 5$ & \\
\hline & CGI & $87 \pm 7$ & 0.09 & $83 \pm 8$ & 0.85 & $86 \pm 8$ & 0.76 & $85 \pm 5$ & 0.45 \\
\hline & CG2(CMI) & $76 \pm 8$ & 0.02 & $74 \pm 9$ & 0.006 & $78 \pm 9$ & 0.004 & $76 \pm 6$ & 0.001 \\
\hline \multirow[t]{3}{*}{ Flow } & MALS & $308 \pm 66$ & & $306 \pm 86$ & & $277 \pm 55$ & & $297 \pm 50$ & \\
\hline & CGI & $316 \pm 64$ & 0.72 & $311 \pm 72$ & 0.84 & $317 \pm 98$ & 0.21 & $314 \pm 57$ & 0.37 \\
\hline & CG2(CMI) & $285 \pm 61$ & 0.29 & $260 \pm 65$ & 0.06 & $240 \pm 65$ & 0.10 & $262 \pm 52$ & 0.06 \\
\hline \multirow[t]{3}{*}{ Velocity } & MALS & $39 \pm 4$ & & $38 \pm 5$ & & $39 \pm 5$ & & $39 \pm 4$ & \\
\hline & CGI & $40 \pm 4$ & 0.36 & $37 \pm 3$ & 0.47 & $38 \pm 5$ & 0.59 & $38 \pm 3$ & 0.90 \\
\hline & CG2(CMI) & $39 \pm 4$ & 0.82 & $33 \pm 4$ & 0.005 & $34 \pm 4$ & 0.006 & $35 \pm 3$ & 0.01 \\
\hline
\end{tabular}

Abbreviations: MALS, median arcuate ligament syndrome; CGI, control group I (normal intestinal circulation); CG2, control group 2; CMI, chronic mesenteric ischemia.

improved. One dimension (self-care) was not affected prior to intervention and remained unchanged.

\section{Discussion}

To our knowledge, this is the first study to investigate the transmucosal microcirculation in patients with MALS using a gastroscopy assisted LDF and VLS. The results of this study support the hypothesis that MALS has an ischemic etiology. We observed a significantly lower transmucosal oxygen saturation in patients with MALS compared to healthy individuals at baseline. Further, a borderline significant increase in transmucosal oxygen saturation was observed after the intervention. In addition, an increase in $\mathrm{rHb}$ and flow after the laparoscopic surgical

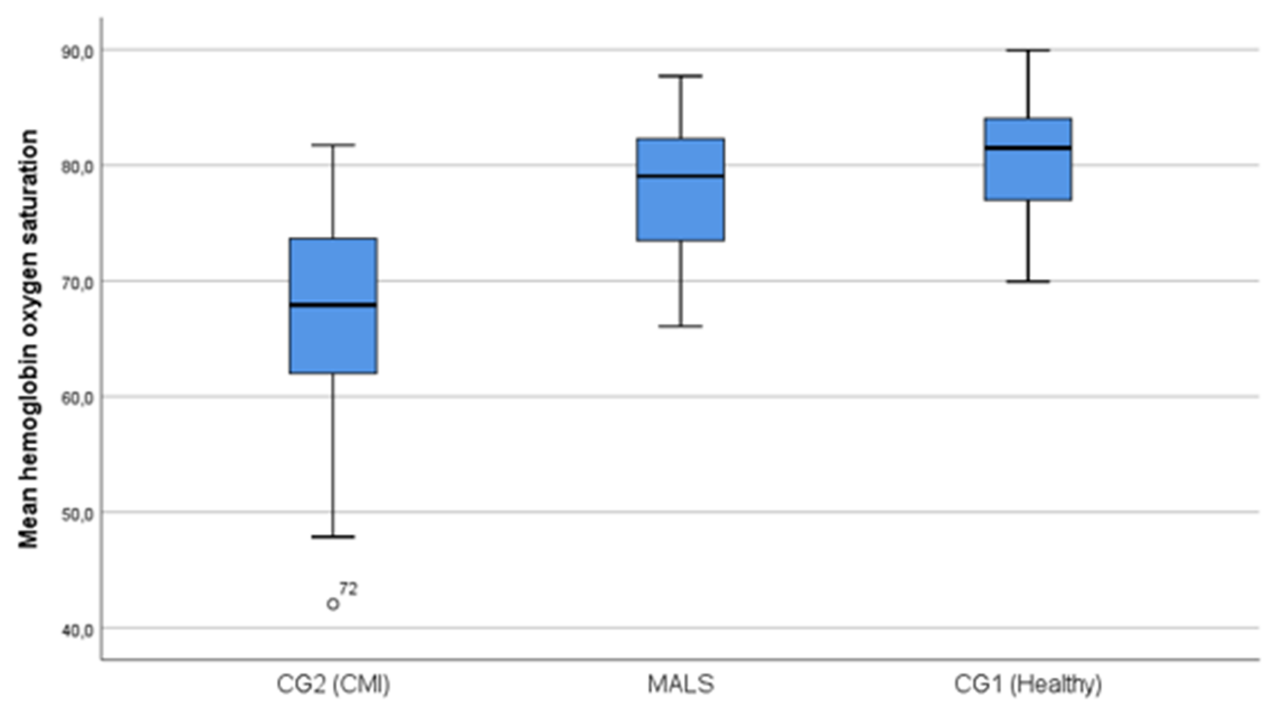

Figure 4 Box-plot of preoperative combined arterial and venous oxygen saturation in CGI $(n=38)$, CG2 (CMI, $n=32)$ and patients with MALS ( $n=I I)$. The thick black line represents the median, the blue box represents the 25-75th percentile and the bars are minimum and maximum points (excluding outliers). Abbreviations: CGI, control group I; CG2, control group 2; CMI, chronic mesenteric ischemia; MALS, median arcuate ligament syndrome. 


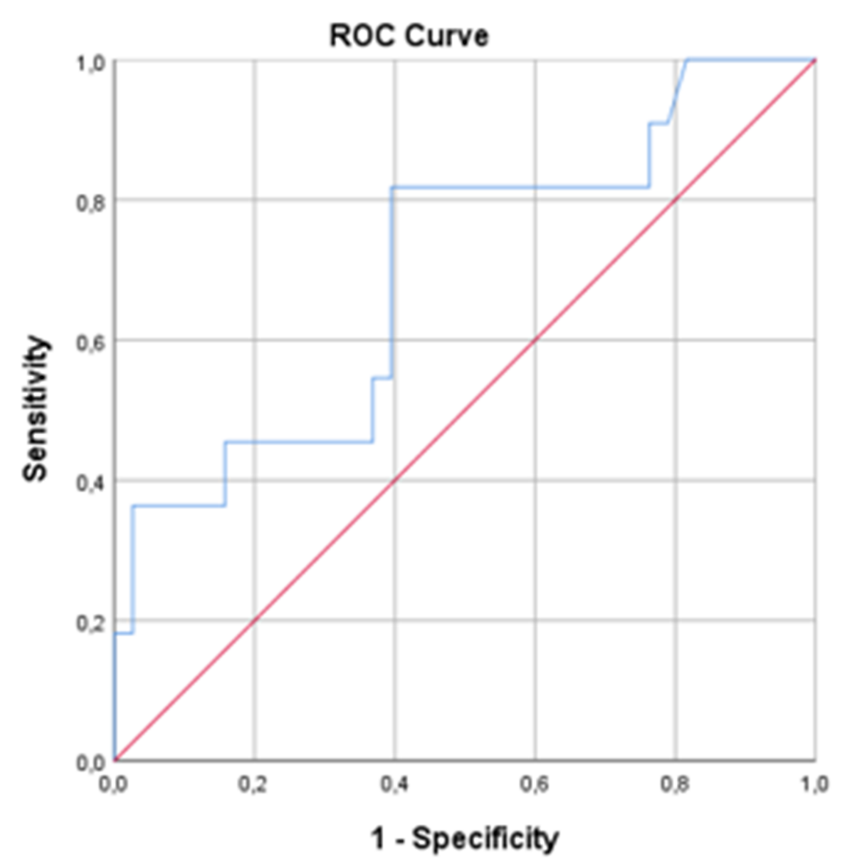

Diagonal segments are produced by ties.

Figure 5 ROC curve of a mean of all measurements of saturation in both the stomach and duodenum in patient with MALS $(n=I I)$ compared to CGI $(n=38)$. Abbreviations: ROC, receiver operated characteristics; MALS, median arcuate ligament syndrome; CGI, control group I; AUC, area under the curve; CMI, chronic mesenteric ischemia.

intervention suggests an improvement in the local perfusion.

Two main hypotheses are postulated to explain the pathophysiology of MALS. ${ }^{5}$ The prevailing theory is that the symptoms are a result of ischemia due to insufficient oxygen delivery, either by means of direct compression of the vessel or due to a neuropathic component leading to
Table 4 Combined Arterial and Venous Oxygen Saturation $\left(\mathrm{SO}_{2}\right)$, Relative Hemoglobin Amount $(\mathrm{rHb})$, Flow and Velocity Before and After Laparoscopic Decompression of the Celiac Artery in Patients with Median Arcuate Ligament Syndrome

\begin{tabular}{|l|l|l|l|}
\hline Variables $(\mathbf{n}=\mathbf{I} \mathbf{I})$ & & Mean \pm SD & p-value \\
\hline $\mathrm{SO}_{2}$ & $\begin{array}{l}\text { Preoperative } \\
\text { Postoperative }\end{array}$ & $\begin{array}{l}76.6 \pm 6.2 \\
81.0 \pm 3.7\end{array}$ & 0.05 \\
\hline $\mathrm{rHb}$ & $\begin{array}{l}\text { Preoperative } \\
\text { Postoperative }\end{array}$ & $\begin{array}{l}83.8 \pm 4.7 \\
85.9 \pm 3.5\end{array}$ & 0.28 \\
\hline Flow & Preoperative & $299.9 \pm 49.8$ & 0.65 \\
& Postoperative & $313.45 \pm 52.6$ & \\
\hline Velocity & Preoperative & $38.47 \pm 4.2$ & 0.35 \\
& Postoperative & $37.4 \pm 4.1$ & \\
\hline
\end{tabular}

splanchnic vasoconstriction. The reduction in blood flow through the CA could cause a steal effect from the SMA, leading to small bowel ischemia. The other theory is that a neuropathic compression of the vessel may lead to inflammation and irritation of sympathetic pain fibers causing pain, much like in the case of other nerve compression diseases, such as carpal tunnel. ${ }^{6}$ The celiac plexus is a hub for abdominal visceral afferent nerve fibers with pain sensation. Blocking the transmission of pain sensation through this hub has been performed with the injection of anesthetic agents for relief of intractable pain associated with malignant disease and chronic pancreatitis. Limited data on its efficacy are available. One review reports a favorable outcome of the procedure with pain relief in $72 \%$ (celiac plexus neurolysis) of the patients with

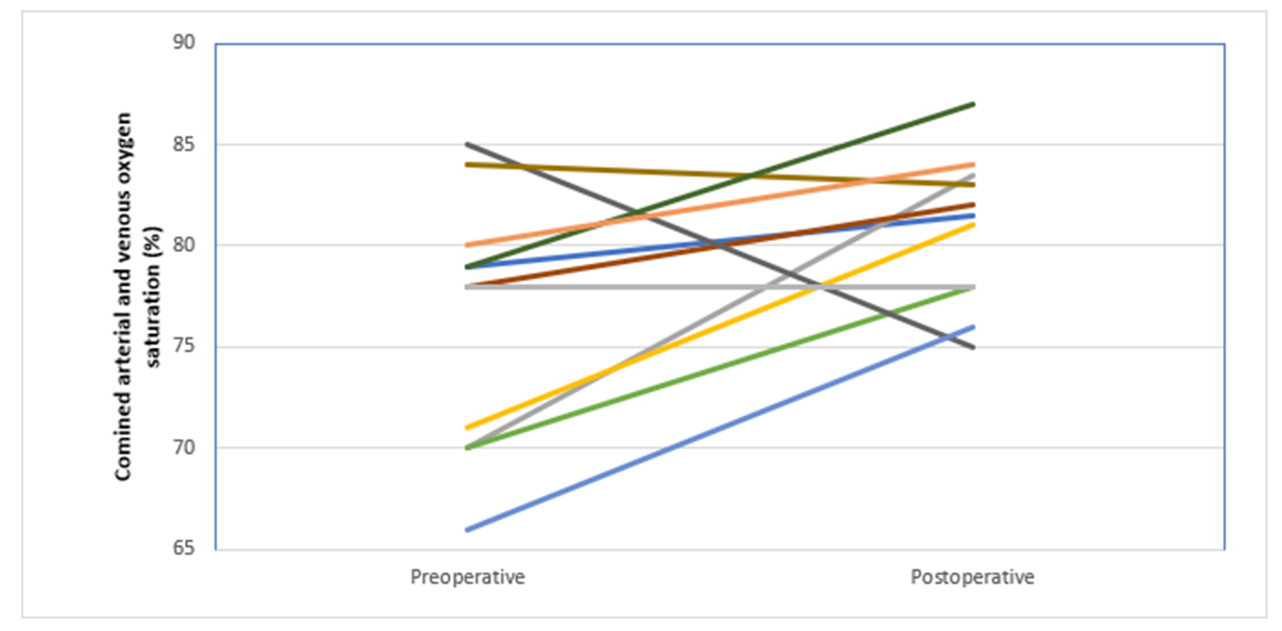

Figure 6 Combined arterial and venous oxygen saturation (\%), before and after ( $n=11, p=0.05)$ laparoscopic decompression of the CA in patients with MALS. Each line represents one patient.

Abbreviations: MALS, median arcuate ligament syndrome; CA, celiac artery. 


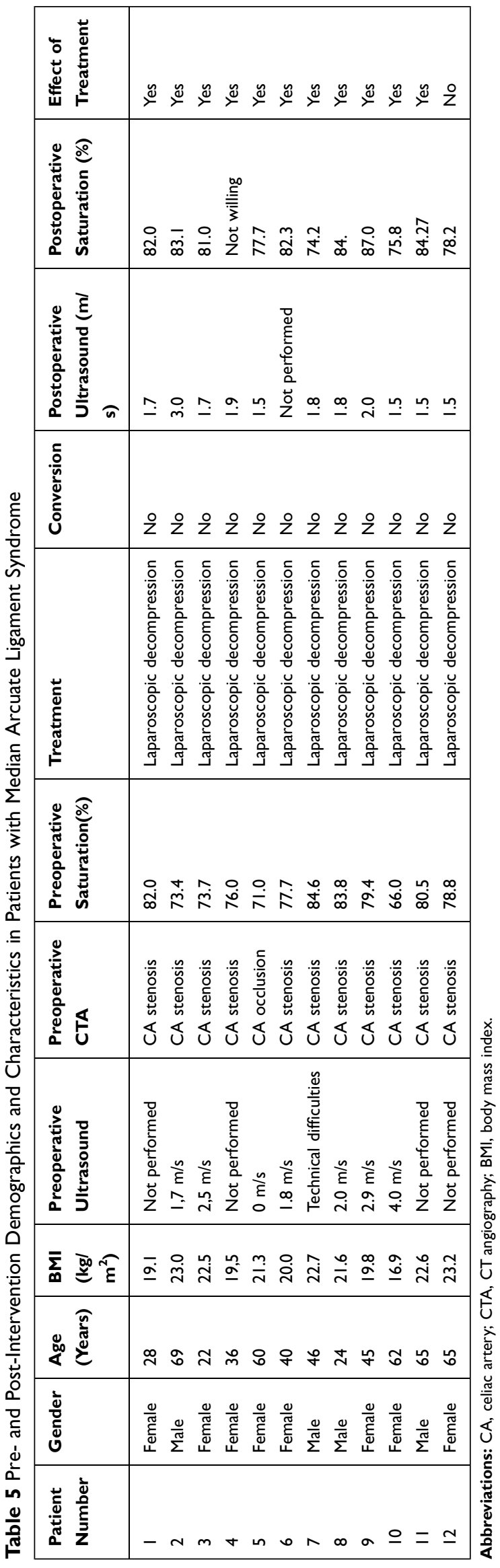

pancreatic cancer and 51\% (celiac plexus block) of the patients with chronic pancreatitis. ${ }^{17}$

The excellent collateral circulation of the bowel and the cyclic change in the rate of stenosis of the celiac artery should intuitively permit an adequate supply to the microcirculation in a resting, fasting state. To unveil significant ischemic changes to the microcirculation in patients diagnosed with MALS was not anticipated.

In our previous study, CMI patients had significantly reduced microcirculation compared to control individuals, suggesting that VLS could play an essential part in the diagnostic process. The sensitivity of VLS $\left(\mathrm{SO}_{2}+\mathrm{rHb}\right)$ for diagnosing CMI was $97 \%$ and specificity $79 \%$. VLS appeared to be a more sensitive diagnostic test for ischemia than LDF. As ischemic changes could be masked by the intermittent nature of the flow reduction in patients with MALS, LDF variables could possibly become more apparent in periods of increased metabolic demand, such as after a meal or during physical exercise with a redistribution of blood flow to skeletal muscles. In the study with GET on patients with MALS, physical exercise is used as a stress test and resulted in the detection of ischemic changes in these patients. However, in a recently published study with VLS after luminal feeding in patients with CMI, mucosal oxygen saturation increased in both healthy individuals and CMI patients, and the provocation provided no discriminative ability towards the diagnosis of CMI. ${ }^{18}$ This study had limitations, as natural digestion is merely approximated, and measurements were performed after an expected peak in postprandial hyperemia. Only one study has previously, successfully, utilized a functional test to evaluate the microcirculation in these patients, where an increase in $\mathrm{pCO}_{2}$ was found in patients with MALS when applying GET. ${ }^{8}$ GET is a rather cumbersome technique and is no longer available on the market.

In our study, the transmucosal oxygen saturation increased, and the velocity declined after decompression of the CA in eight of eleven patients with symptom relief (one patient declined the second upper endoscopy). No change in saturation was detected in the patient without clinical response.

Both LDF (velocity) and VLS (saturation) were able to detect changes after intervention. Although $\mathrm{SO}_{2}$ seems to be the best variable for the detection of ischemia in the microcirculation in these patients, a combination of the two methods allows a more detailed understanding of the physiological changes in the microcirculation before and after surgical intervention. 
The improvement in the transmucosal saturation in the patients with symptomatic improvement may help to conclude a successful laparoscopic decompression of the CA. In our study, we were not able to reproduce the diagnostic accuracy of VLS during upper endoscopy with regards to CMI. With a cut-off $80 \% \mathrm{SO}_{2}$, the sensitivity is $82 \%$ but according to our results, sensitivity and specificity are still too low for this test to be utilized for patient selection.

A common view is that stenosis of a single mesenteric artery is insufficient to lead to mesenteric ischemia. However, several recent studies, including our own on CMI, suggest that ischemia can develop even with single vessel pathology. ${ }^{10,19,20}$

Follow-up with duplex ultrasound after laparoscopic decompression shows normalization of velocities in all controlled patients except one.

Although we present a material over 36 months from Norway's largest hospital, a relatively small number of patients were included. MALS patients were significantly younger than healthy individuals (CG1) $(\mathrm{p}=0.01)$ and had a lower BMI. In comparison to CMI patients (CG2), MALS patients had a significantly lower prevalence of hypertension, diabetes mellitus, intermittent claudication and ischemic heart disease. Furthermore, patients with MALS and CMI were examined with CT angiography, and healthy controls were examined with color duplex ultrasound.

Laparoscopic decompression for MALS was first reported in 2000 and is now the treatment of choice in this patient group. ${ }^{4,21,22}$ The procedure requires a small operating field and is well fitted for laparoscopic surgery. The approach used in our institution has proven to be safe and to date without complications. None of the patients in this study have reported any adverse events or worsening of their preoperative symptoms.

In total, $92 \%$ of the treated patients reported symptom relief, suggesting that our patient selection was adequate. A correlation between the severity of a stenosis and outcome after surgical decompression has been described, with better clinical outcome found in patients with $\geq 70 \%$ stenosis. ${ }^{23}$ Our patients had $\geq 50 \%$ stenosis. However, as this is an exclusion diagnosis, a test with higher specificity is warranted to identify patients who might benefit from surgery.

\section{Conclusion}

Gastroscopy assisted VLS may detect perioperative changes in the transmucosal $\mathrm{SO} 2$ in patients with MALS. Laparoscopic decompression is a safe and effective treatment of patients with MALS. Improvement in transmucosal SO2 after laparoscopic decompression may support a possible ischemic etiology in patients with MALS.

\section{Data Sharing Statement}

Individual participant data that underlie the results reported in this article, after deidentification (text, tables and figures), will be made available at the conclusion of the ongoing study on chronic mesenteric ischemia at Oslo University Hospital, in 2022 and be available for three years. In addition to this, the study protocol will be available. Data will be shared with investigators whose proposed use of the data has been approved by an independent review committee identified for this purpose. Proposals should be directed to M.D. PhD Syed Sajid Hussain Kazmi, syekaz@ous-hf.no, project leader. To gain access, data requestors will need to sign a data access agreement.

\section{Author Contributions}

All authors, STB, NS, AWM, JOS, JH, and SSHK made a substantial contribution to conception and design, acquisition of data, analysis, and interpretation of data; took part in drafting the article and revising it critically. All authors gave final approval of the version to be published; and agree to be accountable for all aspects of the work.

\section{Disclosure}

The authors report no conflicts of interest in this work.

\section{References}

1. Lipshutz B. A composite study of the coeliac axis artery. Ann Surg. 1917;65(2):159. doi:10.1097/00000658-191702000-00006

2. Horton KM, Talamini MA, Fishman EK. Median arcuate ligament syndrome: evaluation with CT angiography. Radiographics. 2005;25 (5):1177-1182. doi:10.1148/rg.255055001

3. Bjorck M, Koelemay M, Acosta S, et al. Editor's choice - management of the diseases of mesenteric arteries and veins: clinical practice guidelines of the European Society of Vascular Surgery (ESVS). Eur J Vasc Endovasc Surg. 2017;53(4):460-510. doi:10.1016/j.ejvs.2017. 01.010

4. Kim EN, Lamb K, Relles D, Moudgill N, DiMuzio PJ, Eisenberg JA. Median arcuate ligament syndrome-review of this rare disease. JAMA Surg. 2016;151(5):471-477. doi:10.1001/jamasurg.2016.0002

5. Bech FR. Celiac artery compression syndromes. Surg Clin North Am. 1997;77(2):409-424. doi:10.1016/S0039-6109(05)70558-2

6. Weber JM, Boules M, Fong K, et al. Median arcuate ligament syndrome is not a vascular disease. Ann Vasc Surg. 2016;30:22-27. doi:10.1016/j.avsg.2015.07.013

7. Early DS, Ben-Menachem T, Decker GA, et al. Appropriate use of GI endoscopy. Gastrointest Endosc. 2012;75(6):1127-1131. doi:10.1016/ j.gie.2012.01.011

8. Mensink PB, van Petersen AS, Kolkman JJ, Otte JA, Huisman AB, Geelkerken RH. Gastric exercise tonometry: the key investigation in patients with suspected celiac artery compression syndrome. $J$ Vasc Surg. 2006;44(2):277-281. doi:10.1016/j.jvs.2006.03.038 
9. Urbanavičius L, Pattyn P, Van de Putte D, Venskutonis D. How to assess intestinal viability during surgery: a review of techniques. World J Gastrointest Surg. 2011;3(5):59-69. doi:10.4240/wjgs.v3. i5.59

10. Berge ST, Safi N, Medhus AW, et al. Gastroscopy assisted laser doppler flowmetry and visible light spectroscopy in patients with chronic mesenteric ischemia. Scand $J$ Clin Lab Invest. 2019;79:1-9.

11. Friedland S, Benaron D, Coogan S, Sze DY, Soetikno R. Diagnosis of chronic mesenteric ischemia by visible light spectroscopy during endoscopy. Gastrointest Endosc. 2007;65(2):294-300. doi:10.1016/j. gie.2006.05.007

12. Fajer S, Cornateanu R, Ghinea R, Inbar R, Avital S. Laparoscopic repair of median arcuate ligament syndrome: a new approach. $J \mathrm{Am}$ Coll Surg. 2014;219(6):e75-e78. doi:10.1016/j.jamcollsurg.2014.08. 009

13. Jimenez JC, Harlander-Locke M, Dutson EP. Open and laparoscopic treatment of median arcuate ligament syndrome. J Vasc Surg. 2012;56(3):869-873. doi:10.1016/j.jvs.2012.04.057

14. Moneta GL, Lee RW, Yeager RA, Taylor LM Jr, Porter JM. Mesenteric duplex scanning: a blinded prospective study. $J$ Vasc Surg. 1993;17(1):79-86. doi:10.1016/0741-5214(93)90011-A

15. Forst T, Hohberg C, Tarakci E, Forst S, Kann P, Pfützner A. Reliability of lightguide spectrophotometry $(\mathrm{O} 2 \mathrm{C})$ for the investigation of skin tissue microvascular blood flow tissue oxygenation supply in diabetic and nondiabetic subjects. J Dia Sci Tech. 2008;2 (6):1151-1156

16. Rabin R, Charro F. EQ-SD: a measure of health status from the EuroQol Group. Ann Med. 2001;33(5):337-343. doi:10.3109/ 07853890109002087
17. Kaufman M, Singh G, Das S, et al. Efficacy of endoscopic ultrasound-guided celiac plexus block and celiac plexus neurolysis for managing abdominal pain associated with chronic pancreatitis and pancreatic cancer. J Clin Gastroenterol. 2010;44(2):127-134. doi:10.1097/MCG.0b013e3181bb854d

18. van Dijk LJD, Harki J, van Noord D, et al. Detection of mesenteric ischemia by means of endoscopic visible light spectroscopy after luminal feeding. Gastrointest Endosc. 2019;89(1):94-102. doi:10. 1016/j.gie.2018.07.024

19. Mensink P, Van Petersen A, Geelkerken R, Otte J, Huisman A, Kolkman J. Clinical significance of splanchnic artery stenosis. Brit J Surg. 2006;93(11):1377-1382. doi:10.1002/bjs.5481

20. van Noord D, Kuipers EJ, Mensink PB. Single vessel abdominal arterial disease. Best Pract Res Clin Gastroenterol. 2009;23 (1):49-60. doi:10.1016/j.bpg.2008.11.012

21. El-Hayek KM, Titus J, Bui A, Mastracci T, Kroh M. Laparoscopic median arcuate ligament release: are we improving symptoms? J Am Coll Surg. 2013;216(2):272-279. doi:10.1016/j.jamcollsurg.2012.10. 004

22. Roayaie S, Jossart G, Gitlitz D, Lamparello P, Hollier L, Gagner M. Laparoscopic release of celiac artery compression syndrome facilitated by laparoscopic ultrasound scanning to confirm restoration of flow. J Vasc Surg. 2000;32(4):814-817. doi:10.1067/mva.2000.10 7574

23. Cienfuegos JA, Estevez MG, Ruiz-Canela M, et al. Laparoscopic treatment of median arcuate ligament syndrome: analysis of long-term outcomes and predictive factors. J Gastrointest Surg. 2018;22(4):713-721. doi:10.1007/s11605-017-3635-3
Vascular Health and Risk Management

\section{Publish your work in this journal}

Vascular Health and Risk Management is an international, peerreviewed journal of therapeutics and risk management, focusing on concise rapid reporting of clinical studies on the processes involved in the maintenance of vascular health; the monitoring, prevention and treatment of vascular disease and its sequelae; and the involvemen of metabolic disorders, particularly diabetes. This journal is indexed on PubMed Central and MedLine. The manuscript management system is completely online and includes a very quick and fair peerreview system, which is all easy to use. Visit http://www.dovepress. com/testimonials.php to read real quotes from published authors. 